\title{
Mamíferos do Pleistoceno Superior de Afrânio, Pernambuco, nordeste do Brasil
}

\author{
Mammals from Upper Pleistocene of Afrânio, Pernambuco, northeast \\ of Brazil
}

\author{
Fabiana Marinho Silva ${ }^{\text {ab }}$, César Felipe Cordeiro Filgueiras ${ }^{\text {ac }}$, Alcina Magnólia Franca \\ Barreto $^{\text {ad }}$, Édison Vicente Oliveira ${ }^{\text {ae }}$ \\ aniversidade Federal de Pernambuco \\ b fabirk26@yahoo.com.br, ${ }^{\mathbf{c}}$ cesar.filgueiras@ufpe.br, ${ }^{\mathbf{d}}$ alcina@ufpe.br, edison.vicente@ufpe.br
}

\begin{abstract}
RESUMO
Os mamíferos pleistocênicos são encontrados com frequência em toda a região Nordeste do Brasil. Os fósseis em geral ocorrem em tanques, lagoas, terraços fluviais, cavernas e ravinas. No estado de Pernambuco são registradas ocorrências de mamíferos pleistocênicos em 38 municípios. Neste trabalho, foram estudados aspectos taxonômicos e tafonômicos de paleofauna, preservada em lagoas da bacia do riacho Caboclo, tributário do rio São Francisco, em Afrânio, Pernambuco, Brasil. A pesquisa envolveu levantamento bibliográfico, cartográfico, trabalhos de campo e laboratoriais. Mais de 1.250 ossos, dentes e osteodermos foram estudados. A associação fossilífera é monotípica, poliespecífica, com os graus de fragmentação e desgaste variando em quatro classes. Os ossos foram preservados por conservação da composição química original, permineralização e substituição por calcita e por calcita magnesiana. Foi identificada uma diversificada fauna distribuída em cinco ordens (Tardigrada, Cingulata, Notoungulata, Proboscidea e Perissodactyla), sete famílias (Megatheriidae, Mylodontidae, Dasypodidae, Glyptodontidae, Toxodontidae, Gomphotheriidae e Equidae) com os taxa: Eremotherium laurillardi, Mylodonopsis ibseni, Panochthus greslebini, Holmesina paulacoutoi, Hoplophorus euphractus, Stegomastodon waringi, Toxodon platensis, equídeo e gliptodontideo indeterminados. Foram registrados pela primeira vez em Pernambuco os gêneros Hoplophorus e Mylodonopsis. A paleofauna é predominantemente herbívora, de um paleoambiente de savana ou cerrado. Através da datação por Luminescência Opticamente Estimulada (LOE) de sedimentos depositados em camadas acima dos fósseis, estimou-se uma idade mais antiga do que $11.300 \pm 2.000$ anos para a última fase de ocupação da megafauna na região.
\end{abstract}

Palavras-chave: Pleistoceno; Mamíferos; Taxonomia; Tafonomia; Afrânio.

\begin{abstract}
Pleistocene mammals are frequently found in the northeastern region of Brazil. In general the fossils occur in tanks, lagoons, fluvial terrains, caves and ravines. In the state of Pernambuco, pleistocene mammals happen to occur in 38 municipalities. In this work, taxonomic and taphonomic aspects of paleofauna - which was found preserved in the lagoons from Caboclo bay, tributary of São Francisco river, Afranio, Pernambuco, Brazil - were studied. Research involved cartographic and bibliographic survey, besides field and laboratorial work. More than 1.250 bones, teeth and osteoderms were studied. The fossiliferous association is monotypical, polispecific to the fragmentation and with damage degree with variations in 4 classes. The bones were preserved by conservation of the original chemical composition, permineralization and calcite and magnesian calcite substitution. A diversified fauna was identified distributed in five orders: Tardigrada, Cingulata, Notoungulata, Proboscidea and Perissodactyla, seven families: Megatheriidae, Mylodontidae, Dasypodidae, Glyptodontidae, Toxodontidae, Gomphotheriidae e Equidae, with the following taxa: Eremotherium laurillardi, Mylodonopsis ibseni, Panochthus greslebini, Holmesina paulacoutoi, Hoplophorus euphractus, Stegomastodon waringi, Toxodon platensis, undetermined equid and gliptodontid. It was registered for the first time in the state of Pernambuco the genera Hoplophorus and Mylodonopsis. The paleofauna is predominantly herbivorous, from a savannah paleoenvironment or sparse bushy and forested vegetation areas. Ages of Optically Stimulated Luminescence (OSL) of deposited sediments, in upper layers was estimated an age older than $11.300 \pm 2.000$ years for the last megafauna occupation phase in the region.
\end{abstract}

Keywords: Pleistocene; Mammals; Taxonomy; Taphonomy; Afrânio. 


\section{Introdução}

No Brasil, ocorrem fósseis de mamíferos em todas as regiões (Bergqvist \& Almeida 2004), e em todos os estados do Nordeste são encontrados mamíferos pleistocênicos. Esses achados estão registrados na literatura desde o início do século XX por pesquisadores como Branner (1902), Vidal (1946), Rosado (1957), Carvalho (1968), Damasceno (1973) e Rolim (1971, 1974, 1981), que contribuíram de forma significativa com o desenvolvimento da paleontologia na região. Os fósseis em geral ocorrem em pequenas áreas sedimentares pleistocênicas como tanques naturais (cacimbas), lagoas, terraços fluviais, cavernas e ravinas (Araújo Junior \& Porpino 2007, Bergqvist et al. 2004, Santos 2001, Cartelle 1992, Rolim 1981, Paula Couto 1953).

A maior parte dessas descobertas ocorreu de forma acidental por moradores da região, quando buscavam alternativas para acumular a água das chuvas, para o período da estiagem, escavando tanques preenchidos por sedimento e, nesse sedimento, muitas vezes eram encontrados os fósseis (Branner 1902, Vidal 1946, Rolim 1974, Mabesoone \& Rolim 1973, Rolim 1981, Silva et al. 2006).

No estado de Pernambuco são registradas ocorrências de mamíferos pleistocênicos em 38 municípios com a presença de oito ordens Tardigrada Lathan \& Davis, 1795; Cingulata Illiger, 1811; Proboscidea Illiger, 1811; Notoungulata Roth, 1903; Carnivora Bowdich, 1821; Artiodactyla Owen, 1848; Perissodactyla Owen, 1848; Litopterna Ameghino, 1889 - e 12 gêneros Eremotherium Spillmannm 1948; Panochthus Burmeister, 1866; Glyptodon Owen, 1839; Pampatherium Ameghino, 1880; Holmesina Simpson, 1930; Stegomastodon Pohlig, 1912; Toxodon Owen, 1837; Xenorhinotherium Cartelle \& Lessa, 1988; Hippidion Owen, 1869; Equus Linnaeus, 1758; Hippocamelus Leuckart, 1816; Smilodon Lund, 1842 -, distribuídos em 12 famílias: Megatheriidae Trouessart, 1904; Mylodontidae Ameghino, 1889; Glyptodontidae Burmeister, 1879; Pampatheriidae Paula Couto, 1954; Dasypodidae Gray, 1821; Gomphotheriidae Cabrera, 1929; Toxodontidae Gervais, 1847; Macrauchenidae Gill, 1872; Camelidae Gray, 1821; Cervidae Gray, 1821; Equidae Steinmann \& Doderlein, 1890; Felidae Gray, 1821 (Rolim 1971, 1974, 1981, Silva et al. 2006, Vidal 1946). Predominam no estado as ordens Tardigrada e Proboscidea (Silva et al. 2006).

Este trabalho apresenta o estudo de uma fauna abundante e diversificada de mamíferos pleistocênicos preservada em lagoas de Caboclo, município de Afrânio, Pernambuco, e tem como objetivo estudar os aspectos tafonômicos e taxonômicos de mamíferos pleistocênicos da localidade, contribuindo para a preservação e divulgação do patrimônio paleontológico da região e para o conhecimento sobre a evolução e aspectos paleobiogeográficos do grupo.

\section{2. Área de estudo}

A área de estudo localiza-se no povoado Caboclo, município Afrânio, estado de Pernambuco (08³0'54”S e 4100'18”W), no submédio curso do rio São Francisco (figura 1). Geologicamente, a área está inserida na Província Estrutural da Borborema - porção nordeste da Plataforma Sul Americana (Gomes 2001).

Os jazigos fossilíferos estudados se preservaram em depósitos de lamas e areias que preencheram pequenas lagoas efêmeras, localizadas na meia encosta de planaltos dissecados, que vêm sendo recorrentemente alagadas quando ocorrem as chuvas, por descargas hídricas das cabeceiras da drenagem do riacho Caboclo, afluente do São Francisco, no povoado de Caboclo.

As lagoas Tanque (S 08 $28.904^{\prime}$ e W 040 56.482'), Comprida (S 08 28.654' e W 040 56.274') e Caveira (S 08 $28.046^{\prime}$ e W 040 57.954') foram selecionadas e escavadas parcialmente.

A região apresenta clima tropical semiárido, do tipo BSh de Köppen, com chuvas de verão. Tem vegetação de caatinga, com porte arbustivo a arbustivo-arbóreo ou raramente arbóreo (Beltrão et al. 2005).

\section{Metodologia}

Durante os 15 dias de trabalhos de campo, foram escavadas cinco trincheiras para observações estratigráficas, bioestratinômicas e coleta de fósseis nas lagoas selecionadas, por apresentarem-se com nível freático mais baixo. Também foram analisados fósseis encontrados na superfície das lagoas, exumados por escavações de moradores locais, e da coleção particular do Sr. Albino Lopes.

Os fósseis foram levados para o Laboratório de Paleontologia do Departamento de Geologia da Universidade Federal de Pernambuco (DGEO/UFPE), onde se fez a triagem, limpeza, preparação mecânica, descrição, medidas com paquímetro, identificação, fotografias e tombamento dos exemplares mais diagnósticos para classificação, na Coleção Científica de Macrofósseis do DGEO/CTG/UFPE.

A identificação dos fósseis seguiu Paula Couto (1979), Cartelle (1992), Porpino (1999), Porpino \& Bergqvist (2002) e Prado et al. (2005). Para comparação, utilizou-se o acervo da Coleção Científica de Macrofósseis do DGEO-CTG-UFPE. 


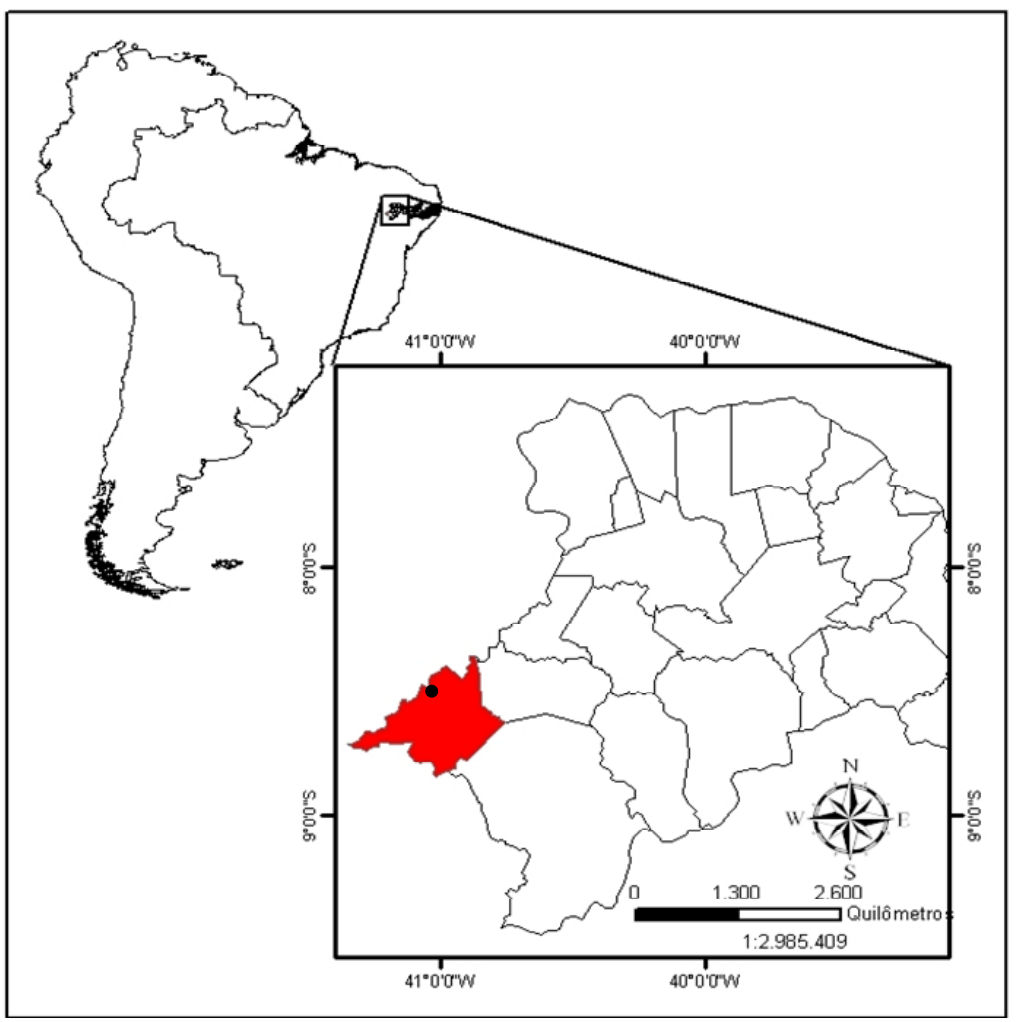

Figura 1: Povoado Caboclo, Afrânio, Pernambuco, Brasil

A datação dos sedimentos por Luminescência Opticamente Estimulada (LOE) foi realizada no Laboratório de Vidros e Datação da Faculdade de Tecnologia de São Paulo - FATEC. Grãos de quartzo foram datados pelo método da regeneração total em intervalo de temperatura de 30 a $475^{\circ} \mathrm{C}$.

\section{Considerações estratigráficas, geocronológicas e tafonômicas}

As lagoas Caveira, Tanque e Comprida apresentaram até 2,4 $\mathrm{m}$ de profundidade e foram preenchidas por cinco camadas de sedimentos siliciclásticos (lama, silte argiloso, silte arenoso, areia síltica e conglomerado), geralmente com estrutura maciça, estando os bioclastos depositados no primeiro estrato (figura 2). Os bioclastos estão dispostos paralelos e perpendiculares ao acamamento, não tendo sido observada orientação definida. Nas lagoas Tanque e Caveira, observou-se uma camada de cerca de $30 \mathrm{~cm}$ de espessura, com fósseis moderadamente empacotados, cimentados por carbonato de cálcio, formando um conglomerado com bioclastos e clastos de seixos, blocos e raros matacões de quartzo, granito e sienitos, que constituem o embasamento das lagoas. Alves (2007) e Silva (2008) descreveram camadas semelhantes a esta em tanques fossilíferos do nordeste brasileiro e interpretaram como calcretes.

Sedimentos depositados em camada acima da concentração de fósseis nas lagoas Tanque e Comprida, datados por Luminescência Opticamente
Estimulada (LOE), apresentaram respectivamente idades de $18.500+2.200$ e $11.300+2.000$ anos. A idade obtida na Lagoa Tanque tem boa coincidência com o último máximo glacial (LGM) e a da Lagoa Comprida correlaciona-se com a passagem do último glacial para o atual Interglacial, Holoceno. É possível estimar uma idade mais antiga do que $11.300 \pm 2.000$ anos para a ocupação desses animais na região.

O material coletado totalizou mais de 1.250 peças, constando de ossos cranianos, pós-cranianos, dentes e um grande número de osteodermos. Parte dos ossos apresentou-se parcialmente articulada e inteira, no entanto, a grande maioria está com diferentes graus de fragmentação e desgaste. Na grande maioria dos casos, a fragmentação precedeu a deposição, evidenciando que houve transporte dos ossos após a morte do animal. Em outros casos, verificou-se que a fragmentação também ocorreu pós-soterramento.

De acordo com o grau e o tipo de fragmentação, os fósseis foram contados e agrupados em quatro classes tafonômicas: 1. inteiro (12); 2. fragmentado com superfície angulosa (194); 3. fragmentado com superfície angulosa e arredondada (915); 4. fragmentado com superfície arredondada (139). A maior parte do material está incluída na classe fragmentada com superfície angulosa e arredondada, sendo possível inferir que houve transporte depois da morte dos animais e provável mistura de esqueletos em tempos diferentes, provavelmente sob fluxo turbulento. 


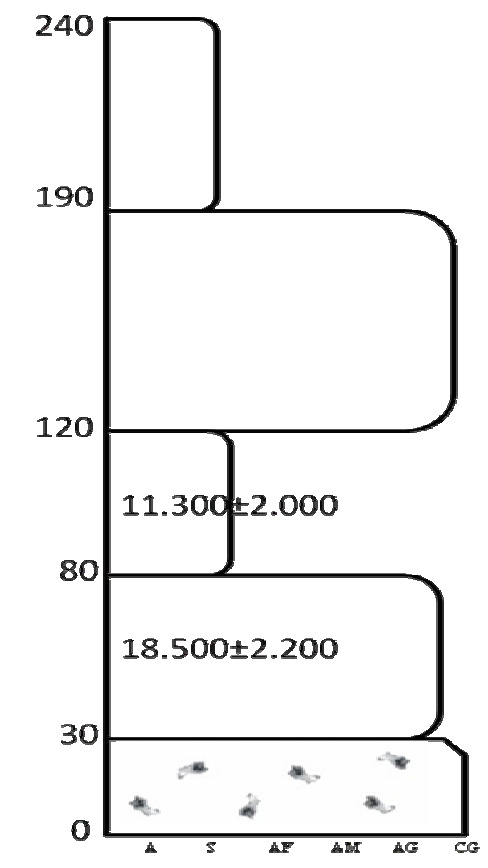

Figura 2: Seção esquemática das trincheiras escavadas em Caboclo, Afrânio, Pernambuco

Os jazigos apresentam concentrações monotípicas (mamíferos) e poliespecíficas (nove taxa). Podem refletir condições de stress ambiental, como possíveis secas, ou ainda, preservação diferencial durante a diagênese, devido à resistência dos ossos, dentes e osteodermos dos megamamíferos. Análises de difratometria de raios-x mostraram preservação dos fósseis por conservação da biomineralização original, permineralização, substituição por calcita e por calcita magnesiana.

\section{Sistemática}

Foram encontrados nove taxa de mamíferos, distribuídos em cinco ordens e sete famílias. A classificação utilizada baseou-se na taxonomia proposta por Paula Couto (1979), Cartelle (1992), Porpino (1999), Porpino \& Bergqvist (2002) e Prado et al. (2005).

Filo CHORDATA
Subfilo VERTEBRATA
Classe MAMMALIA Linnaeus, 1758
Coorte PLACENTALIA (= EUTHERIA) Gill, 1872
Magnaordem EDENTATA Vic D’Azyr, 1792
Superordem XENARTHRA Cope, 1889
Grandordem PHYTOPHAGA Huxley, 1871
Ordem TARDIGRADA Latham \& Davis, 1795
Subordem PILOSA Flower, 1883
Superfamília MEGATHERIOIDEA Gray, 1821
Família MEGATHERIIDAE Trouessart, 1904
Subfamília MEGATHERIINAE Gill, 1872
Gênero Eremotherium Spillmann, 1948

Filo CHORDATA Subfilo VERTEBRATA orte PLACENTALIA (= EUTHERIA) Gill, 1872

Grandordem PHYTOPHAGA Huxley, 1871

Subordem PILOSA Flower, 1883

Superfamília MEGATHERIOIDEA Gray, 1821

Subfamília MEGATHERIINAE Gill, 1872

Gênero Eremotherium Spillmann, 1948
Eremotherium laurillardi (Lund 1842) Cartelle \& Bohórquez, 1982

Idade e paleobiogeografia: Pleistoceno Superior da América do Sul, Central e América do Norte. Pleistoceno Superior do Brasil, Equador, Panamá, Colombia, Venezuela, El Salvador, Honduras, México e Estados Unidos (Cartelle \& Iuliis 2006).

No Brasil são encontrados nos estados do Acre, Amazonas, Rondônia, Alagoas, Bahia, Ceará, Paraíba, Pernambuco, Piauí, Rio Grande do Norte, Sergipe, Goiás, Mato Grosso, Minas Gerais, Rio de Janeiro, São Paulo, Paraná e Rio Grande do Sul (Rolim 1981, Cartelle, 1992).

Espécimes referidos: coleção particular do Sr. Albino Lopes: 1, dentário esquerdo incompleto (figura 3A); 2, dentário esquerdo incompleto; 3, dentário direito incompleto; 4, fragmento de úmero; 5, calcâneo; 6, astrágalo; 7, fragmento de crânio; 8, metatarso.

DGEO-CTG-UFPE: 6531, vértebra cervical incompleta; 6520, 6524, molariformes incompletos; 6522, navicular; 6518, tíbia.

Descrição:

- Os fragmentos dentários esquerdos apresentam a região ventral (bossa) pouco desenvolvida. Em vista oclusal, observam-se quatro molariformes, o $\mathrm{M}_{4}$ menor. $\mathrm{O}$ início da região sinfisiária coincide com o $\mathrm{M}_{1}$, ausência de diastema, cinco forames na face lateral externa (de um dos exemplares), que apresenta uma morfologia convexa; a face lateral interna é suavemente côncava com uma pequena região entre $M_{2}$ e $M_{4}$ reta. Um dos exemplares encontra-se com a região anterior fragmentada até o $\mathrm{M}_{1}$. No exemplar fragmentado de mandíbula direita, estão implantados os $\mathbf{M}_{2}, \mathrm{M}_{3}$ e $\mathbf{M}_{4}$. Fragmento de crânio referente ao osso parietal, bastante fragmentado, sendo possível sua identificação a partir da comparação com exemplar depositado na coleção. Fragmentos de molariformes de formato prismáticos apresentando desgaste das cristas, sem esmalte.

- Uma tíbia com as epífises fragmentadas, diáfise com rachaduras longitudinais e transversais. Um fragmento de úmero direito com crista deltopeitoral presente ao longo da diáfise, epífise distal fragmentada, achatada antero posteriormente, ausência de epífise proximal.

- Um fragmento de vértebra cervical com apenas corpo vertebral completo, projeções ausentes e segunda vértebra cervical (axis) sem projeções; esta apresenta canal medular e vários forames entre o canal medular e o corpo.

- Astrágalos, esquerdo e direito, de tamanhos diferentes com formatos semicirculares, processos odontóides presentes, um deles pouco fragmentado e outro com alto grau de fragmentação, superfícies articulares sustentacular e ectal separadas pelo sulco talis.

- Navicular bem preservado com borda medial convexa e borda lateral côncava, metade dorsal em 
formato de domo semiesférico e metade plantar côncava. Na face distal estão presentes as duas articulações para o astrágalo.

- Calcâneo com superfície dorsal bastante fragmentada, e com as facetas ectal e sustentacular de articulação com o astrágalo presentes e preservadas.

- Metatarso IV com faces articulares para a falange proximal, cubóide e metatarso III bem preservados.

Comentários:

O material referente a Eremotherium laurillardi foi descrito de acordo com o estudado por Cartelle (1992) e Bergqvist et al. (1997) para o Nordeste e assemelha-se com o material encontrado por Rolim (1971), no estado de Pernambuco. Os dentes e mandíbulas, segundo Cartelle (1992), apresentam caráter relevante para descrição da espécie Eremotherium laurillardi, como molariformes de contorno oclusal semelhantes ao dentário, com região ventral (bossa) pouco projetada, diferente do que se observa em Megatherium, que apresenta a mesma região bem desenvolvida. $\mathrm{O}$ astrágalo apresenta característica dos pertencentes a Eremotherium laurillardi, tendo processo odontóide pouco mais acentuado do que os outros representantes da ordem. Eremotherium possuía um regime alimentar herbívoro, alimentava-se de plantas típicas de ambiente de savana com arbustos espinhosos e de cerrado.

Família MYLODONTIDAE Ameghino, 1889 Subfamília MYLODONTINAE Gill, 1872

A

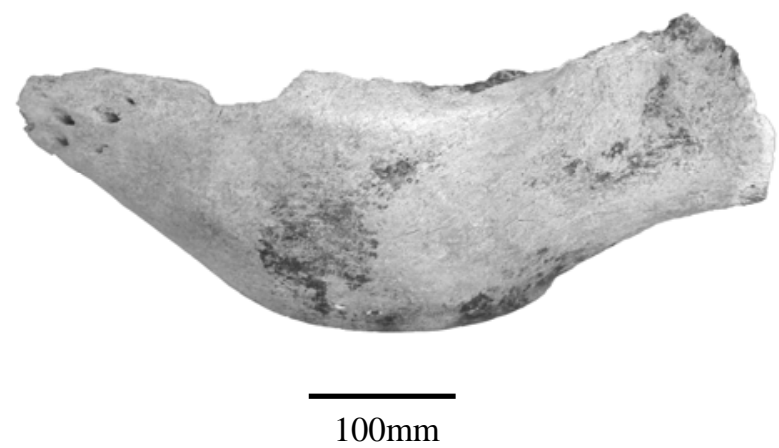

Gênero Mylodonopsis ibseni Cartelle, 1991

Idade e paleobiogeografia: Pleistoceno Superior do Brasil: Bahia, Pernambuco e Minas Gerais (Cartelle 1992).

Espécime referido: DGEO-CTG-UFPE - 6525, fragmento de dentário esquerdo (figura 3B).

Descrição:

Fragmento de dentário esquerdo com face lateral convexa e lingual côncava. A face ventral apresenta quatro alvéolos. Superfície oclusal apresenta três molariformes implantados, ausência de diastema. $\mathrm{M}_{1}$ de formato oval, menor em relação aos outros, mais arredondado mesial do que distalmente. $\mathrm{M}_{2}$ possui, na face lingual, uma concavidade suave. $\mathrm{M}_{3}$ possui face lingual mais estreita e menos sulcada que as outras.

Comentários:

O gênero Mylodonopsis foi descrito em 1991, na Gruta dos Brejões, Bahia, e possui semelhança com o exemplar estudado. O gênero foi descrito por Cartelle (1992) com base em material coletado em Ourolândia, estado da Bahia. A morfologia do dentário juntamente com a morfologia dos dentes mostra que o exemplar deve pertencer a Mylodonopsis ibseni. O estreitamento rostral e a ampla região pré-dentária, presentes no gênero, são indícios de que o alimento seria predominante herbáceo; língua e lábios muito móveis deveriam ter importante papel. Seria uma espécie autóctone, devendo estar inserida em habitat de cerrado.

Figura 3: A - Eremotherium laurillardi, dentário esquerdo incompleto; B - Mylodonopsis sp., fragmento de dentário esquerdo.

Subordem CINGULATA Illiger, 1811 Superfamília GLYPTODONTOIDEA Simpson, 1931

Família GLYPTODONTIDAE Burmeister, 1879 Subfamília SCLEROCALYPTINAE Trouessart, 1898

Tribo PANOCHTINI Simpson, 1945

Gênero Panochthus Burmeister, 1866

Panochthus greslebini Castellanos, 1941
B

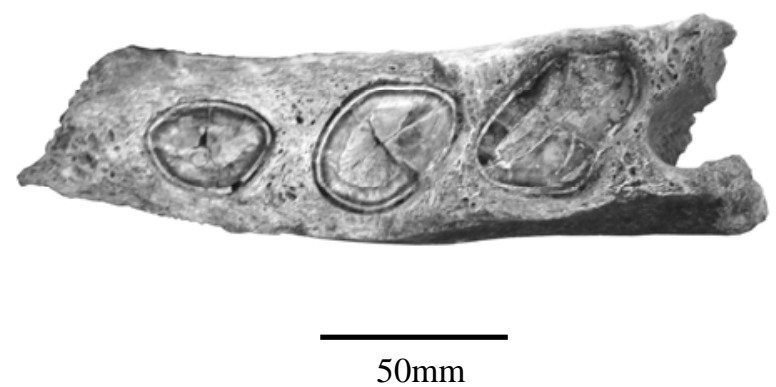


Espécimes referidos: DGEO-CTG-UFPE - 6519, 6521, fragmentos de tubo caudal (figura 4A); 6526, fragmento de carapaça (figura 4B); 6527, 6528, 6529, osteodermos isolados (figura 4C).

Descrição:

O fragmento de tubo caudal exibe um padrão ornamental típico da espécie, com figuras laterais grandes, elevações e superfície enrugada. A superfície interna apresenta vários forames vasculares. $\mathrm{O}$ fragmento de carapaça encontrado faz parte da região dorsal. Composta por cinco osteodermos, um deles com uma figura circular central maior circundada por figurinhas menores, que são separadas por sulcos estreitos. Nos sulcos que separam as figurinhas é possível notar a presença de alguns orifícios pilíferos. A superfície interna apresenta pouca rugosidade e alguns orifícios. Fragmento de carapaça da região dorsal, onde não foi possível observar a superfície interna que está aderida à rocha, com quatro osteodermos. Na superfície externa é possível observar a delimitação entre os osteodermos, pequenas figurinhas separadas por sulcos estreitos e nesses sulcos vários orifícios pilíferos. Foram encontrados nove osteodermos da borda lateral da carapaça, com formato retangular. Estes osteodermos apresentam uma figura circular maior deslocada para a região ventral, circundada por figurinhas periféricas. A superfície interna rugosa apresenta várias perfurações.

Comentários:

Panochthus possui uma das maiores carapaças com osteodermos grandes e poligonais no dorso, menores e mais retangulares na região lateral, formando filas transversais.

O material descrito foi atribuído a Panochthus baseado nas descrições de Cartelle (1992) e Porpino (1999) para material encontrado na Bahia e Rio Grande do Norte, respectivamente. O gênero é encontrado em toda a região Nordeste do Brasil. $P$. jaguaribensis e $P$. greslebini seriam endêmicos. Segundo Porpino \& Bergqvist (2002) P. greslebini difere de $P$. jaguaribensis na espessura da carapaça, com osteodermos mais espessos e o tubo caudal mais achatado dorso-ventralmente e transversalmente alargado. Apresentam a carapaça convexa na região médio-dorsal, ornamentada com placas grossas, grandes e poligonais. Cada placa é ornamentada com numerosas figuras poligonais pequenas e uniformes. As figuras centrais diferem das figuras das placas laterais da carapaça que apresentam uma figura circular maior deslocada para a borda do osteodermo (Paula Couto 1979, Porpino 1999).

\footnotetext{
Subfamília HOPLOPHORINAE Weber, 1928

Tribo HOPLOPHORINI Hoffstetter, 1958

Gênero Hoplophorus Lund, 1839

Hoplophorus euphractus Lund, 1839
}

Idade e paleobiografia: Pleistoceno Superior da América do Sul, Pleistoceno Superior do Brasil, estados do Amazonas, Rondônia, São Paulo, Minas Gerais, Bahia, Ceará, Paraíba, Pernambuco, Piauí e Rio Grande do Norte (Paula Couto 1953, 1979).

Espécime referido: DGEO-CTG-UFPE - 6523, fragmento de carapaça (figura 4D).

Descrição:

Fragmento de carapaça composta por quatro osteodermos de pouca espessura (em torno de 16 $\mathrm{mm}$ ) e formato subcircular. Os osteodermos são ornamentados por uma figura central circundada por figurinhas menores em número de dez. As figuras são separadas por sulcos estreitos e pouco profundos com orifícios pilíferos. Superfície interna lisa apresentando vários orifícios.

Comentários:

Os osteodermos da carapaça foram atribuídos à espécie pela primeira vez em 1939, por Lund, sendo encontrados em cavernas no estado de Minas Gerais. As placas dorsais são semelhantes às de Glyptodon, mas este apresenta um número de figuras periféricas nos osteodermos que varia de seis a nove, e para Hoplophorus é descrito um número de figuras periféricas que varia de nove a onze. Em 1971, Rolim registrou a presença de Hoplophorus sp. no município de Santa Cruz do Capibaribe, mas o material se tratava de osteodermos de Glyptodon - possuía uma figura central circundada por oito figuras menores e apresentava grande espessura. O tamanho da figura central em relação ao das figuras periféricas não constitui um caráter diagnóstico, pois pode variar de acordo com a porção da carapaça a que pertencem os osteodermos. Forames vasculares numerosos, principalmente nos pontos de confluências dos sulcos que dele se irradiam para a periferia da placa. Nos osteodermos dorsais há tendência para formação de duplo círculo de figuras menores em torno das centrais; placas dorsais com superfície áspera. A espessura da carapaça do dorsal é de aproximadamente $17 \mathrm{~mm}$ (Paula Couto 1957, Cartelle 1992).

\section{Subfamília GLYPTODONTINAE Gray, 1869 Gênero cf. Glyptodon Owen, 1839}

Idade e paleobiogeografia: Plioceno e Pleistoceno da América do Sul. No Brasil foi registrada ocorrência nos estados do Rio Grande do Sul, São Paulo, Minas Gerais, Acre e em todos os estados da região Nordeste (Cartelle 1992).

Espécimes referidos: DGEO-CTG-UFPE - 6500, 6502, 6503, 6504, 6505, 6506, 6507, 6508, 6509, 6510 , osteodermos isolados.

Descrição:

O material apresenta superfície externa rugosa, com uma ornamentação externa característica, uma figura central arredondada maior circundada por figuras menores, em número que varia de seis a nove. Ao redor da figura central observa-se um 
sulco mais profundo que os que circundam as figuras ornamentais periféricas. Neste, observa-se a presença de seis forames de grande tamanho coincidindo com o início dos sulcos que circundam as figuras periféricas. Entre eles é possível observar alguns orifícios pilíferos. A espessura variou de 16 a $28 \mathrm{~mm}$.

Comentários:

O material encontrado em Caboclo assemelha-se às características descritas para o material estudado

A

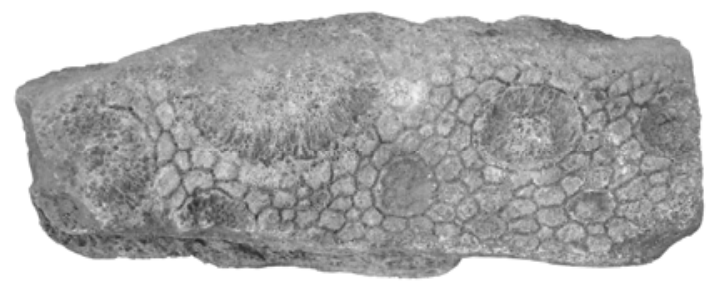

$20 \mathrm{~mm}$

C

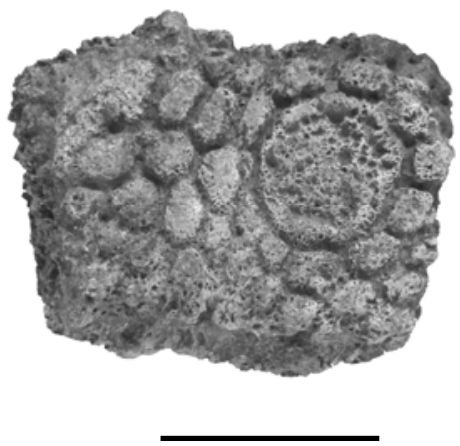

$50 \mathrm{~mm}$ por Cartelle (1992), Porpino (1999) e Porpino et al. (2004). Os osteodermos são espessos e exibem o padrão ornamental em forma de roseta. A figura central arredondada é circundada por figuras periféricas menores, separadas da central por um sulco largo e profundo. São considerados alóctones.
B

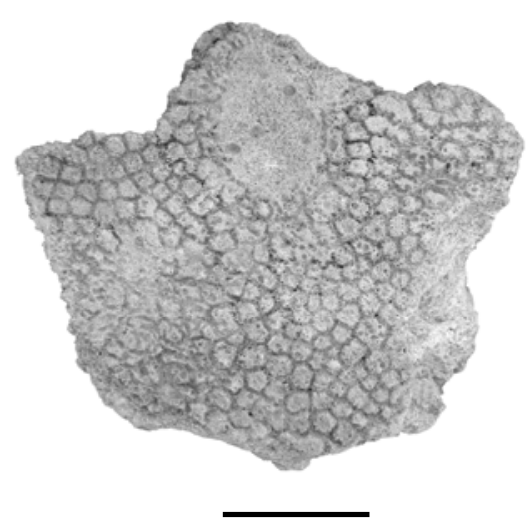

$40 \mathrm{~mm}$

D

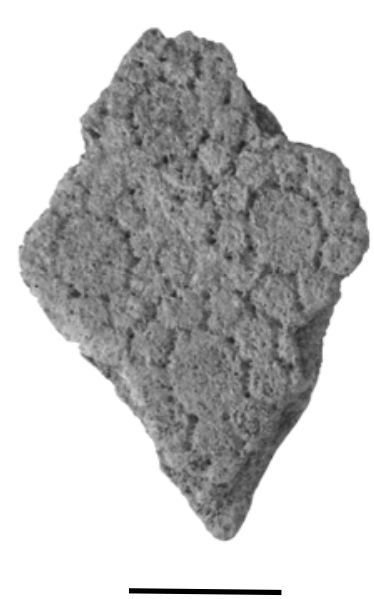

$30 \mathrm{~mm}$

Figura 4: Panochthus greslebini, A. Fragmento de tudo caudal; B. Fragmento de carapaça; C. Osteodermo isolado; D. Hoplophorus euphractus, fragmento de carapaça

Superfamília DASYPODOIDEA Gray, 1821

Família DASYPODIDAE Gray, 1821

Subfamília TOLYPEUTINAE Gray, 1865

Gênero Holmesina Simpson, 1930

Holmesina cf. H. paulacoutoi Cartelle \& Bohórquez, 1985
Idade e paleobiogeografia: Pleistoceno Superior da América do Norte e da América do Sul. Estados Unidos, Brasil, Equador, Argentina, Uruguai e Venezuela. No Brasil são encontrados nos estados da Bahia, Ceará, Paraíba, Piauí, Rio Grande do Norte, Goiás, Minas Gerais, Rio de Janeiro, São Paulo, Rio Grande do Sul (Porpino 1999). 
Espécime referido: DGEO-CTG-UFPE - 6512, 6513, astrágalo direito completo (figura 5A).

Descrição:

Astrágalo em bom estado de preservação. Facetas sustentacular, ectal e para o navicular bem preservadas. As facetas para a articulação com o calcâneo se apresentam com aspecto liso. A faceta para o navicular se apresenta de forma convexa. Presença de um vale longitudinal que separa a crista da tróclea.

Comentários:

Holmesina distingue-se de Pampatherium por características cranianas, dentárias e exoesqueletais (Paula Couto 1979). A espécie foi descrita pela primeira vez no estado de Pernambuco por Alves (2007) através de osteodermos isolados. O astrágalo é bastante semelhante aos descritos para Holmesina majus de Lagoa Santa (Winge 1915), sendo anatomicamente de aspecto mais dasipodídeo do que gliptodontídeo. O astrágalo é identificado como Holmesina por ser mais robusto.

Holmesina era herbívoro, onívoro, pastador; alimentação predominantemente de gramíneas abrasivas, comendo também ovos de outros animais, organismos em decomposição e frutos variados. A alimentação à base de gramíneas abrasivas causava desgaste nos dentes. Habitantes de áreas abertas pouco arborizadas, como savanas.

Ordem PROBOSCIDEA Illiger, 1811

Subordem ELEPHANTOIDEA Osborn, 1921

Família GOMPHOTHERIIDAE Cabrera, 1929

Subfamília ANANCINAE Hay, 1922

Stegomastodon waringi Holland, 1920

= Haplomastodon waringi Holland, 1920

Idade e paleobiogeografia: Plioceno e Pleistoceno da América do Norte e da América do Sul, Pleistoceno da Argentina, Paraguai, Uruguai e Brasil. No Brasil os achados ocorrem nos estados do Acre, Amapá, Amazonas, Rondônia, Alagoas, Bahia, Ceará, Maranhão, Paraíba, Pernambuco, Piauí, Rio Grande do Norte, Sergipe, Goiás, Espírito Santo, Minas Gerais (Simpson \& Paula Couto 1955, Alberdi \& Corona-M. 2005), Rio de Janeiro, São Paulo, Paraná, Rio Grande do Sul (Gadens-Marcon et al. 2006).

Espécimes referidos: coleção particular do Sr. Albino Lopes: 1, molariformes (figura 5B). DGEOCTG-UFPE: 6517, 6532, molariformes.

Descrição:

Molares trilofodontes apresentando pouco desgaste na face oclusiva. Raiz dos exemplares presentes, mas fragmentadas.

Comentários:

Segundo Cartelle (1992) Stegomastodon waringi seria a espécie mais abundante e de distribuição mais ampla entre os mastodontes sul-americanos. Os molariformes apresentam características diagnósticas, como formato retangular, a superfície da coroa com duas séries longitudinais de cúspides cônicas unidas. O estado de preservação do molariforme é bom, mostrando a coroa pouco desgastada e a raiz parcialmente íntegra, desgastados em duplo trevo (Paula Couto 1979).

O tipo de desgaste dos molariformes sugere que sua dentição era adaptada a uma alimentação composta de vegetação de gramíneas abrasivas, ocasionalmente de folhas, brotos de arbustos, típicos de ambientes abertos de cerrado e savana pouco arborizada, e frutos (Paula Couto 1979).

Ordem NOTOUNGULATA Roth, 1903

Subordem TOXODONTIA Owen, 1858

Família TOXODONTIDAE Gervais, 1847

Subfamília TOXODONTINAE Trouessart, 1898

Gênero Toxodon Owen, 1837

Toxodon platensis Owen, 1837

Idade e paleobiogeografia: Pleistoceno da Argentina, Uruguai, Paraguai, Bolívia e Brasil. A primeira ocorrência no Brasil foi citada por Cope, em 1885, para o estado da Bahia. Além da Bahia, os Toxodontidae são encontrados no Acre, Rondônia, Ceará, Paraíba, Piauí, Rio Grande do Norte, Sergipe, Minas Gerais, São Paulo, Paraná e Rio Grande do Sul (Miño Boilini et al. 2006, Paula Couto 1979).

Espécimes referidos: Coleção particular do Sr. Albino Lopes: 1, fragmento de tíbia (figura 5C); 2, pré-molar fragmentado. DGEO-CTG-UFPE: 6530, pré-molar fragmentado.

Descrição:

Dentes pré-molares fragmentados, bilobados com um sulco interlobular; apresenta uma face recoberta inteiramente por esmalte. Tíbia apresenta fragmentação da diáfise, ausência da epífise distal. Superfície de articulação com o fêmur preservada.

Comentários:

Os molares com esmalte, em forma de prisma retilíneo (Paula Couto 1979), de acordo com Vidal (1959) e Paula Couto (1979) são característicos dos pertencentes a Toxodon platensis. O dente molar apresenta faixas de esmalte e dois lóbulos, o anterior de forma elíptica e o posterior marcado por um sulco.

Os toxodontes estão associados a ambientes abertos de pastagens, zonas baixas e inundadas, cursos de água ou proximidades de lagoas, sugerindo um provável hábito anfíbio (Paula Couto 1979).

Ordem PERISSODACTYLA Owen, 1848

Subordem HIPPOMORPHA Wood, 1937

Superfamília EQUOIDEA Hay, 1902

Família EQUIDAE Gray, 1821

Subfamília EQUINAE Steinmann \& Doderlein, 1890

Material: Dente de equídeo em calcrete, com a superfíce oclusal desgastada (figura 5D).

Comentários:

A identificação do material em nível genérico e até específico seria possível através das 
características observadas na superfície oclusal do dente, que no material coletado encontra-se com desgaste acentuado. É possível apenas determinar que o exemplar pertence à subfamília Equinae de acordo com material descrito por Paula Couto (1944). No estado de Pernambuco estão registradas

\section{A}

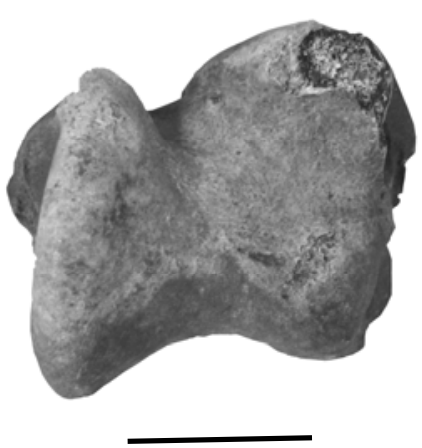

$50 \mathrm{~mm}$

C

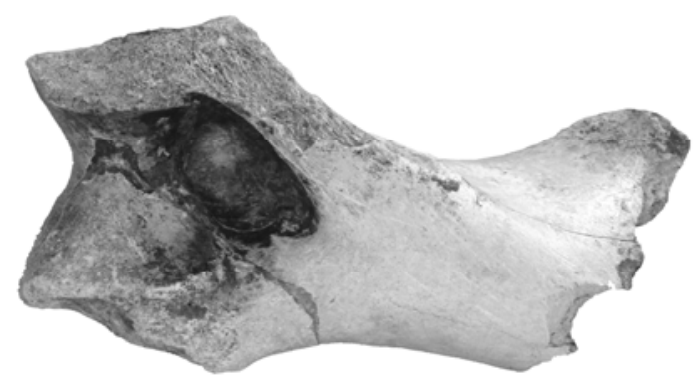

$70 \mathrm{~mm}$ ocorrências de material fossilífero pertencente a Hippidion e Equus (Rolim 1981).

A dentição dos Equinae é adaptada à alimentação à base de vegetais. Segundo Scherer \& Stock da Rosa (2003), são indicativos de ambientes de planície aberta, clima frio e úmido.
B

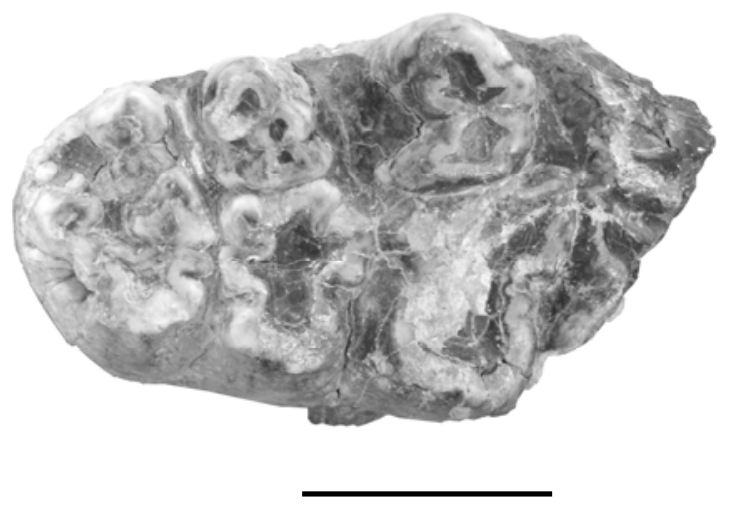

$70 \mathrm{~mm}$

D

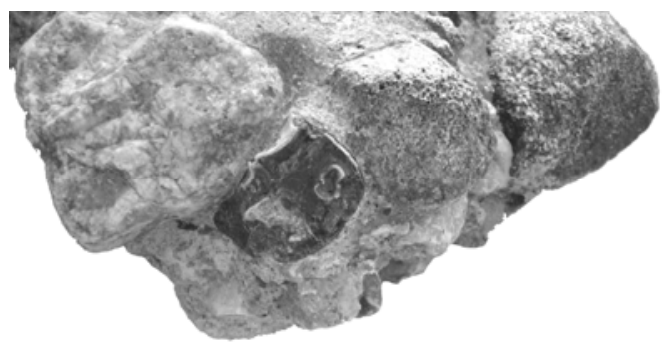

Figura 5: A. Holmesina paulacoutoi, astrágalo direito completo; B. Stegomastodon waringi, molariforme; C. Toxodon platensis, fragmento de tíbia; D. Equídeo indeterminado, dente

\section{Conclusões}

A região de Caboclo, município de Afrânio, Nordeste do Brasil, atualmente sob clima semiárido e vegetação de caatinga, tem grande potencial para estudo de mamíferos pleistocênicos, pela quantidade de depósitos e pela diversidade de megamamíferos descritos aqui, pela primeira vez.

Os jazigos fossilíferos preservaram-se em lamas e areias que preencheram pequenas lagoas efêmeras, localizadas na meia encosta de planaltos dissecados que vem sendo até o presente recorrentemente alagadas, por chuvas torrenciais junto a cabeceiras da drenagem de afluentes do rio São Francisco. Por isso, foram moradores locais, quando escavavam procurando água durante as estiagens da década de 1980, os descobridores dos fósseis.
Os jazigos formam concentrações monotípicas poliespecíficas, devendo refletir condições de stress ambiental, como secas, ou mesmo uma preservação diferencial durante diagênese, devido à resistência dos ossos, dentes e osteodermos dos megamamíferos.

Análises de difratometria de raios- $x$ mostraram preservação dos fósseis por conservação da biomineralização original, permineralização, substituição por calcita e por calcita magnesiana.

A associação fossilífera encontrada é monotípica, poliespecífica. Os bioclastos estão fragmentados e distribuídos em quatro classes de tamanho, indicativo de transporte hidráulico diferenciado após a morte do animal.

A fauna da região apresenta uma boa diversidade de espécies, incluídas em cinco ordens: Tardigrada, 
Cingulata, Notoungulata, Proboscidea e Perissodactyla; e sete famílias: Megatheriidae, Mylodontidae, Glyptodontidae, Dasypodidae, Toxodontidae, Gomphotheriidae e Equidae, representadas pelos taxa Eremotherium laurillardi, Panochthus greslebini, Holmesina paulacoutoi, Toxodon platensis, Stegomastodon waringi, Hophophorus euphractus, Mylodonopsis ibseni, equídeo e um gliptodontídeo indeterminados.

Pode ter havido na paleofauna a predominância dos gliptodontídeos do gênero Panochthus, devido ao grande número de osteodermos e tubos caudais encontrados.

Foram registradas para o Estado de Pernambuco pela primeira vez as espécies Hoplophorus euphractus, a partir de fragmento de carapaça, e Mylodonopsis ibseni, por um fragmento de mandíbula.

A fauna encontrada é predominantemente de herbívoros. O paleoambiente em que esses animais viviam era característico de áreas abertas de savana e cerrado, com vegetação arbustiva e arbórea, contrastando com as condições ambientais atuais da região de Afrânio, onde predomina a caatinga hiperxerófila, existente sob temperatura média de $24,8^{\circ} \mathrm{C}$

É possível estimar uma idade mais antiga do que $11.300+2.000$ anos para a última fase de ocupação desses animais na região.

\section{Agradecimentos}

Agradecemos ao Conselho Nacional de Desenvolvimento Científico e Tecnológico CNPq, Processo 555.951/2006-5, pelo financiamento da pesquisa e pela concessão da Bolsa de Mestrado.

\section{Referências}

Alberdi M. T., Corona-M E. 2005. Revisión de los gonfoterios en el Cenozóico tardío de México. Revista Mexicana de Ciencias Geológicas, 22 (2): 246:260.

Alves R.S. 2007. Os mamíferos pleistocênicos de Fazenda Nova, Brejo da Madre de Deus, Pernambuco: aspectos tafonômicos, taxonômicos e paleoambientais. Dissertação de Mestrado. Pós-Graduação em Geociências, Centro de Tecnologia e Geociências, Universidade Federal de Pernambuco, 140p.

Araújo Junior H.I., Porpino K.O. 2007. Mamíferos fósseis da Fazenda Lágea Formosa, São Rafael, Rio Grande do Norte, Brasil: Interpretações paleoecológicas. In: VIII Congresso de Ecologia do Brasil. Boletim de resumos. Anais, p. 1-2.

Beltrão B.A., Mascarenhas J.C., Miranda J.L.F., Junior L.C.S., Galvão M.J.T., Pereira N.P. 2005. Projeto cadastro de fontes de abastecimento por água subterrânea. Diagnóstico do município de Afrânio, Estado de Pernambuco. Recife: CPRM/PRODEEM, 11p.

Bergqvist L.P., Gomide M., Cartelle C., Capilla R. 1997. Faunas-locais de mamíferos pleistocênicos de Itapipoca/Ceará, Taperoá/Paraíba e Campina Grande/Paraíba. Estudo Comparativo, Bioestratinômico e Paleoambiental. Revista Universidade Guarulhos Geociências, 2(6): 23-32

Bergqvist L.P., Almeida E.B. 2004. Biodiversidade de mamíferos fósseis brasileiros. Revista Universidade de Guarulhos - Geociências, 9(6): 54-68.
Bergqvist L.P., Abuhid V.S., Giudice G.M.L. 2004. Mamíferos. In: Carvalho, I. S. Paleontologia. Interciência. 2. ed., v. 1. p. 833-861.

Branner J.C. 1902. On the occurence of fossil remains of mammals in the interior of the States of Pernambuco and Alagoas, Brazil. The American Journal of Science, 13: 133137

Cartelle C. 1992. Edentata e megamamíferos herbívoros extintos da Toca dos Ossos (Ourolândia, BA, Brasil). Tese de Doutorado. Programa de Pós-Graduação em Morfologia, Universidade Federal de Minas Gerais, 516p.

Cartelle C., De Iuliis G. 2006. Eremotherium laurillardi (Lund) (Xenarthra, Megatheriidae), the Panamerican giant ground sloth: taxonomic aspects of the ontogeny of skull and dentition. Journal of Systematic Paleontology, 4 (2): 199209.

Carvalho C. 1968. Considerations on the pleistocenic fauna of "Lagedo da Escada". Arquivos Instituto de Antropologia v.3, n.1: 1-8.

Damasceno J.M. 1973. Ocorrência de Toxodon platensis Owen, 1840 em Olho d’Água da Escada, distrito de Baraúna, município de Mossoró, Rio Grande do Norte. Arquivos do Instituto de Antropologia Câmara Cascudo - Paleontologia, n. $1: 1-18$.

Gadens-Marcon G.T., Ferigolo J., Ribeiro A.M. 2006. O registro dos Proboscidea para o Quaternário do Estado do Rio Grande do Sul. In: I Semana acadêmica dos alunos de pósgraduação em geociências. Resumos, p. 59-61.

Gomes H.A. 2001. Geologia e recursos minerais do Estado de Pernambuco. Brasília: CPRM/DIEDIG/DEPAT. 198, mapas escala 1:1.500.000.

Mabesoone J.M., Rolim J.L. 1973. Quaternário do Nordeste oriental brasileiro. Estudos Sedimentológicos, 3-4: 89-130.

Miño Boilini, A. R., Cerdeño, E. \& Bond, M. 2006. Revisión del género Toxodon Owen, 1837 (Notoungulata: Toxodontidae) en el Pleistoceno de las provincias de Corrientes, Chaco y Santa Fe, Argentina. Revista Española de Paleontologia, 21 (2), 93-103.

Paula Couto C. 1944. Sobre a presença dos gêneros Hippidion e Toxodon Owen, no Pleistoceno do Rio Grande do Sul. Rio de Janeiro, Boletim do Museu Nacional/Ministério da Educação e Saúde, Geologia, 2. 12p.

Paula Couto C. 1953. Paleontologia brasileira de mamíferos. Instituto Nacional do Livro, Rio de Janeiro. 516p

Paula Couto C. 1957. Sobre um gliptodonte do Brasil. Divisão de Geologia e Mineralogia,DNPM, Boletim, 165: 1-37 p.

Paula Couto C. 1979. Tratado de paleomastozoologia. Academia Brasileira de Ciências, Rio de Janeiro, 590p.

Porpino K.O. 1999. Estudo dos Cingulata (Xenarthra, Mammalia) fósseis depositados no Museu Câmara Cascudo, Natal - RN. Dissertação de Mestrado. Instituto de Geociências, Universidade Federal do Rio de Janeiro, 138p.

Porpino K.O., Bergqvist L.P. 2002. Novos achados de Panochthus (Mammalia, Cingulata, Glyptodontoidea) no Nordeste do Brasil. Revista Brasileira de Paleontologia. 4: 51-62.

Porpino K.O., Santos M.F.C.F., Bergqvist L.P. 2004. Registro de mamíferos fósseis no Lajedo de soledade, Apodi, Rio Grande do Norte, Brasil. Revista Brasileira de Paleontologia, 7 (3): 349-358.

Prado J.L., Alberdi M.T. Azanza B., Sánchez B., Frassinetti D. 2005. The pleistocene Gomphotheriidae (Proboscídea) from South América. Quaternary International, 126-128: 21-30.

Rolim J.L. 1971. Sobre alguns mamíferos fósseis de Lagoa da Pedra - Município de Santa Cruz do Capibaribe Pernambuco. Estudos Geológicos, 1: 1-19.

Rolim J.L. 1974. Calcário secundário com restos fósseis de mamíferos pleistocênicos em Pernambuco. Anais da Academia Brasileira de Ciências, 46 (3/4): 417-422.

Rolim J.L. 1981. Pesquisas de mamíferos pleistocênicos no Nordeste brasileiro. Estudos Geológicos, 4: 57-63.

Rosado V. 1957. A Formação Cacimbas e o Grupo Apodí. Coleção Mossoroense, 34: 1-19.

Santos M.F.C.F. 2001. Geologia e paleontologia de depósitos fossilíferos pleistocênicos do Rio Grande do Norte. 
Dissertação de Mestrado. Centro de Ciências Exatas e da Terra, Universidade Federal do Rio Grande do Norte, 70 p. Scherer C.S., Stock da Rosa A.A. 2003. Um equídeo fóssil do Pleistoceno de Alegrete, RS, Brasil. Pesquisa em Geociências, 30 (2): 33-38.

Silva F.M., Alves R.S., Barreto A.M.F., Sá F.B., Lins e Silva A.C.B. 2006. A megafauna pleistocênica do Estado de Pernambuco. Recife: Estudos Geológicos, 16 (2): 55-66.

Silva J.L.L. 2008. Reconstrução paleoambiental baseada no estudo de mamíferos pleistocênicos de Maravilha e Poço das Trincheiras, Alagoas, Nordeste do Brasil. Tese de Doutorado. Pós-Graduação em Geociências, Centro de Tecnologia e Geociências, Universidade Federal de Pernambuco, $195 \mathrm{p}$.

Simpson G.G., Paula Couto C. 1955. The mastodonts of Brazil. Bul. of the Amer. Mus. of Nat. Hist., New York, 112: 129185.

Vidal N. 1946. Contribuição ao conhecimento da paleontologia do Nordeste brasileiro - Notícia sobre a descoberta de vertebrados pleistocênicos no município de Pesqueira, em Pernambuco. Boletim do Museu Nacional, 6: 15.

Vidal N. 1959. Um Toxodon no Pleistoceno de Pernambuco. Boletim do Museu Nacional, 30: 17.

Winge H. 1915. Jordfundne og nulevende Gumlere (Edentata) fra Lagoa Santa, Minas Gerais, Brasilien. E Museo Lundii, 3 (2) p. 1-321.

Recebido 08 de maio de 2009 Aceito 05 de julho de 2010 